\title{
Beyond the quasistatic approximation: Impedance and capacitance of an exponential distribution of traps
}

\author{
Juan Bisquert* \\ Departament de Física, Universitat Jaume I, 12071 Castelló, Spain \\ (Received 9 November 2007; revised manuscript received 1 March 2008; published 9 June 2008)
}

\begin{abstract}
Electronic carriers in disordered organic and inorganic semiconductor materials, used in electronic, optoelectronic, and photovoltaic devices, are usually affected by an exponential distribution of localized states in the band gap (traps). In this paper we provide a full solution of the relaxation of carriers in traps of such distribution, as a function of frequency and steady-state Fermi level. This includes in a unified treatment both the quasistatic limit, in which the traps modify time constants such as the trap-limited mobility, and the power-law relaxation at high frequency due to detrapping kinetics. We also analyze the combination of trapping with diffusion transport and recombination dynamics in photovoltaic devices. The different features of impedance and capacitance spectra are interpreted and also the analysis of the spectra in order to derive the main material parameters.
\end{abstract}

DOI: 10.1103/PhysRevB.77.235203

PACS number(s): 73.23.-b, 72.20.Jv, 73.61.Ph

\section{INTRODUCTION}

Disordered organic and inorganic materials have raised increasing attention in the last two decades, in relation with applications in devices such as dye-sensitized solar cells (DSCs), ${ }^{1,2}$ plastic solar cells, ${ }^{3,4}$ organic light-emitting diodes (OLEDs), ${ }^{5}$ and organic electronics. ${ }^{6}$ Frequency domain techniques are becoming of widespread used in the study of these materials and devices, since they provide an insight both on fundamental electronic processes and global device properties. Impedance and capacitance spectroscopy of devices s $^{7,8}$ using such materials normally requires to consider a distribution of states in energy. Energy disorder is common for band-gap states of electronic carriers in disordered semiconductors, both for nanostructured metal oxides used in DSC (Refs. 9 and 10) and for organic conductors used in thin-film transistors (TFT) ${ }^{11-13}$ OLEDs, ${ }^{8,14}$ and organic solar cells. ${ }^{15}$ Especially in organic materials, deep traps are reactive sites as they are either radical cations or anions and can play a role in the degradation of organic molecules, such as polyphenylvinylene polymers. However, the degradation occurs at time scales of thousands of hours in the most stable systems. An important factor is therefore the presence of impurities which can also act as trapping sites, extrinsic traps, which can greatly influence the stability of the system. Also in ionic materials such as Li battery cathodes, energetic inhomogeneity has been found. ${ }^{16}$

The exponential distribution of states seems to play a key role in many of the mentioned systems. For example, such distribution is routinely obtained as a chemical capacitance in electrochemical measurements of nanostructured $\mathrm{TiO}_{2}$ (Refs. 17 and 18) and the exponential density of states (DOS) also explains the observed variation of electron diffusion coefficient in DSC using multiple trapping arguments. ${ }^{9,10}$ The exponential DOS is also often employed in connection with organic conductor materials., ${ }^{8,12-15}$ Closely connected to such a distribution is the anomalous diffusion model ${ }^{19}$ that so far seems to be the best approach for interpretation of diffusion of guest ions in transitionmetal oxides..$^{20,21}$
Most of the work presented in the literature for the interpretations of these systems uses a multiple trapping model, where the effect of traps is to reduce the mobility and increase the recombination time by a factor of the free to trapped charge. This approach, which dates back to Rose, ${ }^{22,23}$ is formulated as a quasistatic approximation in Refs. 24 and 25 and has been widely used in the DSC area. ${ }^{10,26}$ Similar approaches to trap-limited mobility are well known in the field of crystalline organic electronic devices ${ }^{27}$ and amorphous organic semiconductors. ${ }^{8,11}$ This approximation is quite useful since it allows us to treat the trap distribution simply as an additional charge, which follows the relaxation of the free charge in the system, so that the time constants in the system can be easily deduced using the kinetic model of the trap-free system. However, any relaxation of the trapped carriers is assumed to be very fast and is neglected from the beginning. However, if the trap distribution indeed exists, one should be able to observe the relaxation of trappingdetrapping kinetics, provided that one measures in the appropriate frequency domain. A direct measurement of trap kinetics is very important for tailoring the materials with required properties for device operation.

There exist classical results on dispersive (power-law) behavior of the photoconductivity and mobility in time transient experiments, which is due completely to the trapping kinetics. $^{28,29}$ Therefore, one can switch from the regime, where the traps remain in equilibrium, to that in which they dominate the transient response..$^{30}$ In this paper we provide a full solution of the relaxation of the exponential distribution of traps, which unifies both regimes of behavior in a single framework, in the frequency domain.

It is well known that in semiconductor devices, each trap level provides a series $R C$ circuit. ${ }^{31}$ The capacitance $C_{\text {trap }}$ is the chemical capacitance of a localized state ${ }^{32}$ and relates to the equilibrium occupancy of the trap $\bar{f}$ (with $0 \leq f \leq 1$ ) as $C_{\text {trap }} \propto \bar{f}(1-\bar{f})$. It should be emphasized that $C_{\text {trap }}$ is related to the variation of the occupation of the trap with respect to the quasi-Fermi level. So this capacitance has nothing to do with a standard dielectric capacitance and is more related to the chemical capacitance, ${ }^{17,33}$ also known as accumulation 


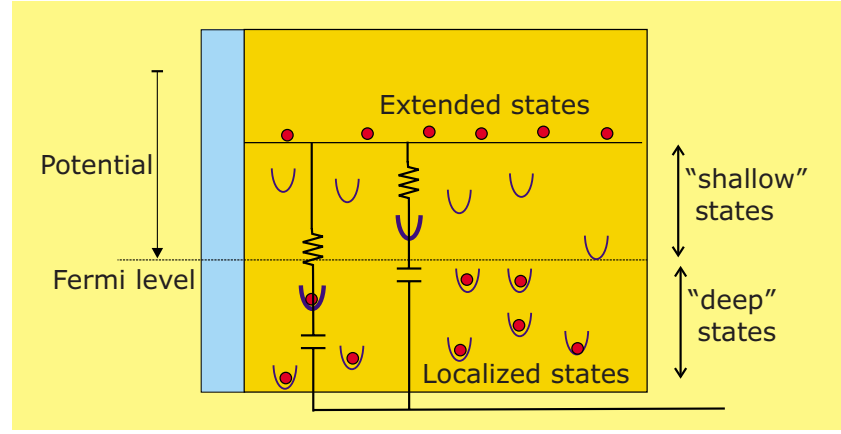

FIG. 1. (Color online) The scheme shows a semiconductor where the Fermi level can be controlled with external potential. The semiconductor has both extended states (e.g., conduction-band levels) and localized (band-gap) levels. The Fermi-level marks approximately the separation between occupied and empty localized levels, which applies in an exponential distribution of traps. The equivalent circuit for two specific levels (one occupied, another one empty) is shown. For each level, the resistance corresponds to the thermal release of electron to the extended levels, and the capacitance corresponds to the variation of equilibrium occupancy of the level under variation of Fermi level, i.e., a chemical capacitance. The extended states also possess a chemical capacitance, though this is not shown. The characteristic frequencies of the equivalent circuits of occupied and empty levels have different properties, as discussed in the text, and are denoted as "deep" and "shallow," respectively.

capacitance ${ }^{34}$ or redox capacitance. ${ }^{35}$ On another hand the resistance $R_{\text {trap }}$ contains the kinetic factor for detrapping. Both values depend on the occupancy of the level, and the characteristic trap frequency is given by

$$
\omega_{t}=\frac{1}{R_{\text {trap }} C_{\text {trap }}} .
$$

The value of $\omega_{t}$ determines the relaxation properties of each level in the distribution. The properties of simple trap combinations in the frequency domain are illustrated in Appendix. We have discussed in detail in a previous paper the frequency-dependent capacitance in a two-state model, ${ }^{36}$ and this model has been successfully applied in experiments of ion insertion in metal oxides. ${ }^{16}$ Using a similar method we provide here a full solution of the capacitance and impedance of the exponential DOS. Since the characteristic time for detrapping depends exponentially on the distance between a trap level and the transport level, the exponential distribution introduces a very large set of time scales. Furthermore, the trap response is largely variable depending on the occupancy of the trap. Therefore, it is required to establish the steady state of the DOS, at given Fermi level, as indicated in Fig. 1, and then to calculate the frequency-dependent capacitance $C^{*}(\omega)$, by integration of the capacitance of the different levels, and the associated impedance, $Z(\omega)=1 / i \omega C^{*}(\omega)$. By summation, we give here a closed expression for the capacitance $C^{*}(\omega)$ of the whole distribution in a good approximation.

Very often, in experimental spectra, several frequency effects due to distinct phenomena (e.g., energy disorder and geometry inhomogeneity ${ }^{16}$ ) have to be deconvoluted. For the interpretation of experimental data, it is very useful to have an understanding, in terms of analytical models, of the different spectral features in separate segments of the frequency window of measurement. Therefore, we will develop analytic approximations to the whole response of the exponential distribution of traps. The shape of spectra and different behaviors obtained at low and high frequencies will be discussed in detail, as well as the dependence of parameters on steady-state conditions, in order to assist the interpretation of experimental data.

The paper is divided into two main parts. Section II treats the relaxation of a continuous distribution of traps in the frequency domain, as shown schematically in Fig. 1. The goal of this section is to find out the local effects caused by the trapping-detrapping phenomena when all the states of the distribution are considered simultaneously. Section III describes an approach to spatially extended device modeling, by considering diffusion trapping in a restricted layer, and the effect of the trap distribution on recombination dynamics in the frequency domain, mainly in relation with photovoltaic systems such as DSC. We expect that these results will find application in a wider variety of systems. In this work we restrict our attention to systems in which the Fermi level can be considered homogeneous across the active device layer. We plan to apply the present developments in highly inhomogeneous systems such as TFTs and OLEDs in a future work. Another interesting development left for the future is to apply a similar method to the Gaussian distribution that is also widely used in organic semiconductors. ${ }^{25,37}$

\section{TRAP MODEL}

Figure 1 shows a basic scheme of the model discussed in this paper. The scheme shows a piece of semiconductor, both with a transport level (extended states), and localized states in the band gap. The traps are assumed to be connected (by trapping-detrapping kinetics) to the transport level, which communicates with the outer contacts. In the scheme, we indicate that the applied potential governs the Fermi level (a specific realization of this is shown in Fig. 8; see also Ref. 2). Therefore, we assume that the local Fermi level can be fixed at a value $E_{F}$.

The measurement involves two levels of perturbation. The first is the steady-state value of the potential. This fixes the occupation of the traps and the free-carrier concentration. More generally, if the traps also serve as recombination centers, they are normally not in thermal equilibrium with the conduction-band carriers, and they require separate Fermi levels. ${ }^{38,39}$ However, in the present work we do not treat such situation, and the traps are conservative with respect to number of carriers. The second level is a small harmonic perturbation. The latter causes the dynamic response of the traps and facilitates the analysis of experimental systems by looking at the spectral features of the capacitance and impedance. In essence the frequency response of a trap is a $R C$ series circuit, as shown in Fig. 1 for two traps and discussed in Appendix. However, there is a great difference of trap response according to their steady-state occupancy. The empty 

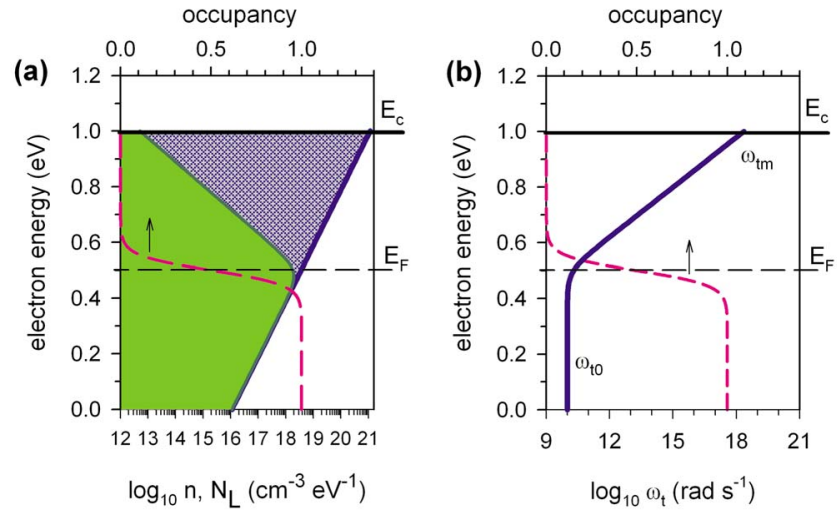

FIG. 2. (Color online) (a) Energy diagram showing the thermal occupation at temperature $T=300 \mathrm{~K}$ of an exponential DOS in the band gap. $E_{F}$ is the Fermi level of electrons and $E_{c}$ is the conduction-band energy. The clear (green) shaded region indicates the band-gap states that are occupied with electrons, and the dark (blue) shaded region indicates empty levels, as determined by the Fermi-Dirac distribution function (red dashed line). (b) The trap frequencies as a function of energy. The parameters used in the calculation: $T_{0}=800 \mathrm{~K}, N_{c}=10^{20} \mathrm{~cm}^{-3}, N_{t}=10^{20} \mathrm{~cm}^{-3}$, and $\beta$ $=10^{-10} \mathrm{~cm}^{2} \mathrm{~s}^{-1}$.

traps readily capture electrons, while the full traps do not. More exactly, the occupation of electron traps above the Fermi is well approximated by the Boltzmann distribution. Below the Fermi level, traps are almost fully occupied, but the density of vacant traps (holes) also obeys the Boltzmann distribution. The occupations are quantitatively shown in Fig. 2(a) in the energy axis. The aim of the following development is to obtain the characteristic spectra of the combined relaxation of all the traps in the exponential distribution.

\section{A. Kinetic equations}

The model is formulated assuming the extended states at the level $E_{c}$ (conduction-band edge), with effective density $N_{c}$ and number density of carriers $n_{c}$. With respect to the Fermi level $E_{F}$, we have

$$
n_{c}=N_{c} e^{\left(E_{F}-E_{c}\right) / k_{B} T},
$$

where $k_{B}$ is Boltzmann's constant and $T$ the temperature. In addition, we assume a distribution of traps $g(E)$, with occupancy $f_{t}(E)$, where $E$ is the energy; see the diagram in Fig. 2(a) that illustrates the exponential distribution. The number of density of carriers in trap states is

$$
n_{t}=\int_{E_{v}}^{E_{c}} g(E) f_{t}(E) d E .
$$

For the sake of brevity we omit the argument of $f_{t}$ in the following. The kinetic equations are

$$
\frac{\partial n_{c}}{\partial t}=-\frac{\partial n_{t}}{\partial t}=-\int_{E_{v}}^{E_{c}} g(E) \frac{\partial f_{t}}{\partial t} d E
$$

$$
\frac{\partial f_{t}}{\partial t}=\beta n_{c}\left[1-f_{t}\right]-\varepsilon f_{t} .
$$

Here, $\beta$ is the time constant for electron capture, usually given in terms of the thermal velocity of the electrons and capture cross section,

$$
\beta=v \sigma_{n} .
$$

In steady state, at given Fermi level $E_{F}$, we have from Eq.

$$
\bar{f}_{t}=\frac{1}{1+\varepsilon /\left(\beta \bar{n}_{c}\right)}=F\left(E_{t}, E_{F}\right)
$$

in terms of the Fermi-Dirac function,

$$
F\left(E, E_{F}\right)=\frac{1}{1+e^{\left(E-E_{F}\right) / k_{B} T}} .
$$

By detailed balance condition, the time constant for release from the traps at the energy $E_{t}$ is

$$
\varepsilon\left(E_{t}\right)=\beta N_{c} e^{\left(E_{t}-E_{c}\right) / k_{B} T} .
$$

\section{B. Frequency-dependent capacitance of a trap distribution}

The method to obtain the frequency-dependent capacitance is explained in detail in Ref. 36. Let us denote steadystate quantities by $\bar{y}$ and small perturbation quantities by $\hat{y}$. The capacitance is the relation of ac charge $(\hat{Q})$ to voltage $(\hat{V})$,

$$
C^{*}(\omega)=\frac{\hat{Q}}{\hat{V}}=\frac{q}{\hat{V}}\left(\hat{n}_{c}+\hat{n}_{t}\right)=\frac{q}{\hat{V}}\left(\hat{n}_{c}+\int_{E_{v}}^{E_{c}} g(E) \hat{f}_{t} d E\right),
$$

where $q$ is the positive elementary charge. Note that all capacitances $C$ in this section are given per unit volume. The extended states follow instantaneously the variation of applied voltage, which causes a variation of the Fermi level of the free electrons in the conduction band, $-q d V=d E_{F}$. (This assumption involves infinitely fast transport in the extended states and will be relaxed in Sec. III.) Therefore, we can use the relationship

$$
\hat{n}_{c}=\frac{d \bar{n}_{c}}{d \bar{V}} \hat{V}
$$

This last equation can be expressed

$$
\hat{n}_{c}=\frac{C_{0}}{q} \hat{V}
$$

in terms of the chemical capacitance of the extended states, ${ }^{17,33}$

$$
C_{0}=q \frac{d \bar{n}_{c}}{d \bar{V}}=\frac{q^{2}}{k_{B} T} \bar{n}_{c} .
$$

In contrast to Eq. (11), the traps do not follow instantaneously the voltage variation, since each trap only communicates with the conduction band. By solving Eq. (5) for a small perturbation, we obtain 


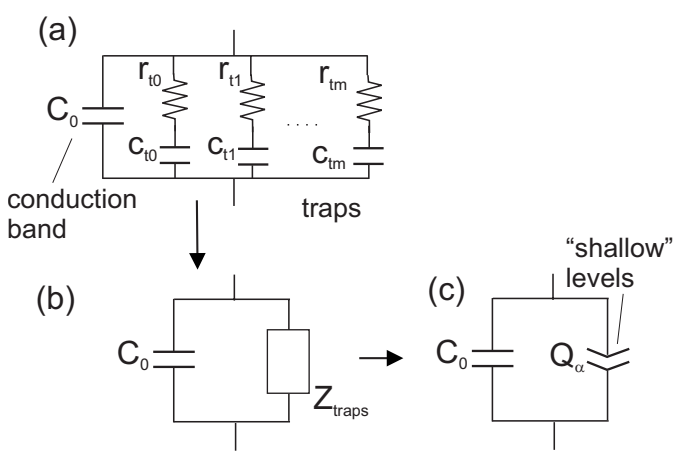

FIG. 3. (a) The equivalent circuit of the conduction-band states (represented by capacitance $C_{0}$ ) and the distribution of traps, each energy interval $d E$ being associated with a $R C$ circuit. (b) The trap impedance is represented by $Z_{\text {traps }}$. (c) Reduction of the impedance of an exponential distribution of traps $Z_{\text {traps }}$ to a much simpler equivalent circuit, valid in the high-frequency domain, as is demonstrated quantitatively in the text. The sum of individual $R C$ elements, each corresponding to a narrow energy interval, over all the shallow levels (above the Fermi level) collapses into a CPE element.

$$
\hat{f}_{t}=\frac{1}{\bar{n}_{c}} \frac{\bar{f}_{t}\left(1-\bar{f}_{t}\right)}{1+i \omega / \omega_{t}} \hat{n}_{c},
$$

where the trap frequency has the expression

$$
\omega_{t}=\frac{\varepsilon}{1-\bar{f}_{t}} .
$$

From Eqs. (12) and (14) we obtain that the trap occupancy depends on the frequency as

$$
\hat{f}_{t}=\frac{q}{k_{B} T} \frac{\bar{f}_{t}\left(1-\overline{f_{t}}\right)}{1+i \omega / \omega_{t}} \hat{V}
$$

Inserting Eqs. (13) and (16) in Eq. (10), we obtain

$$
C^{*}(\omega)=C_{0}+C_{\text {traps }}^{*}(\omega) .
$$

The second term in Eq. (17) is the frequency-dependent capacitance of the trap distribution. It has the form

$$
C_{\text {traps }}^{*}(\omega)=\int_{E_{v}}^{E_{c}} \frac{C_{t}(E)}{1+i \omega / \omega_{t}} d E,
$$

where $C_{t}(E)$ is the equilibrium chemical capacitance per unit energy, ${ }^{32}$

$$
C_{t}(E)=\frac{q^{2}}{k_{B} T} g(E) \bar{f}_{t}\left(1-\bar{f}_{t}\right)
$$

so that $C_{t} d E$ is the capacitance associated to the traps in the interval $d E$. Equation (18) means a parallel connection of all the separate capacitances of the traps, as indicated in Fig. 3.

Integrating Eq. (14) over the energy axis, we can write the relationship between ac modulation of trapped and free carriers as

$$
\hat{n}_{t}=\frac{C_{\text {traps }}^{*}(\omega)}{C_{0}} \hat{n}_{c} .
$$

From Eqs. (18) and (19) a numerical calculation of the frequency-dependent trap capacitance, at given Fermi level, is now possible and will be used below to plot the exact results. As indicated above, we also wish to understand the separate features of the spectra in different ranges of the frequency domain. According to Eq. (9) the levels below $E_{F}$ are almost fully occupied, while the levels above $E_{F}$ are populated following the Boltzmann distribution: $f_{t}$ $=e^{-\left(E-E_{F}\right) / k_{B} T}$ [see Fig. 2(a)]. This makes a great difference for the kinetics of the traps; therefore, a main distinction in the calculation below is made for trap levels above and below the Fermi level. We have denoted them, respectively, as "shallow" and "deep" traps, as indicated in Fig. 1. This is a convenient notation for the purpose of the model that does not imply that either kind of trap is close or far from the transport level in an absolute sense. In addition, the Boltzmann statistics can also be used to describe vacant states below the Fermi level. The use of the Boltzmann statistics, to describe the trap occupancy in suitable domains of the energy axis, is a good approximation for the exponential distribution and other distributions.

However, in other cases, such as the Gaussian distribution when the Fermi level is deep, this approach fails. Indeed the carrier occupation at low carrier density has the form of a Gaussian distribution around $\sigma_{d}^{2} / k_{B} T$ below the center of the DOS (Ref. 37) (see Ref. 25 for a discussion of this question). Thus, the Gaussian distribution requires a separate treatment.

\section{Characteristic trap frequencies}

Let us discuss the dependence of characteristic frequencies [Eq. (15)] on the trap energies using approximations to the Fermi-Dirac distribution $\bar{f}_{t}$. For $E_{t}<E_{F}$, the trap frequency is constant,

$$
\omega_{t 0}=\beta \bar{n}_{c} \approx \beta N_{c} e^{-\left(E_{c}-E_{F}\right) / k_{B} T} .
$$

On another hand, for $E_{t}>E_{F}$ the trap frequency increases with $E_{t}$,

$$
\omega_{t}\left(E_{t}\right) \approx \beta N_{c} e^{-\left(E_{c}-E_{t}\right) / k_{B} T} .
$$

These results are illustrated in Fig. 2(b) and are independent of the distribution $g(E)$, provided that the approximation of the Boltzmann distribution can be used, as already mentioned. The different dependence of $\omega_{t}$ below and above $E_{F}$ makes it a useful step to split the integral in Eq. (18) in two parts,

$$
\begin{aligned}
C_{\text {traps }}^{*}(\omega)= & C_{\text {traps }}^{*}(\omega)+C_{\text {traps }}^{*}(\omega) \equiv \int_{E_{v}}^{E_{F}} \frac{C_{t}(E)}{1+i \omega / \omega_{t}} d E \\
& +\int_{E_{F}}^{E_{C}} \frac{C_{t}(E)}{1+i \omega / \omega_{t}} d E .
\end{aligned}
$$

\section{Capacitance of deep levels}

According to Eq. (21), all the occupied sites bellow $E_{F}$ (which we will call the deep levels) relax with the same 
frequency, $\omega_{t 0}$, which is also the slowest frequency in the distribution. We can immediately infer that the deep levels can be described as a $R C$ series circuit,

$$
C_{\text {deep }}^{*}(\omega)=\frac{C_{\text {deep } 0}}{1+i \omega / \omega_{t}} .
$$

The constant capacitance in Eq. (24) consists simply on the summation of the chemical capacitances over all the levels,

$$
C_{\text {deep } 0}=\frac{q^{2}}{k_{B} T} \int_{E_{v}}^{E_{F}} g(E) \bar{f}_{t}\left(1-\bar{f}_{t}\right) d E .
$$

The resistance in the equivalent circuit of Eq. (24) is (see Appendix)

$$
R_{\text {deep }}=\frac{1}{\omega_{t 0} C_{\text {deep } 0}} .
$$

Since $\omega_{t 0}$ is the slowest frequency in the distribution, we deduce that the observation of the trap relaxation in the impedance measurement will occur only if the measurement frequencies are $\omega>\omega_{t 0}$. If, on the other hand, the measurement is such that $\omega<\omega_{t 0}$, Eq. (18) reduces to a constant capacitance, the chemical capacitance of the localized levels,

$$
C_{\text {traps }}=\frac{q^{2}}{k_{B} T} \int_{E_{v}}^{E_{c}} g(E) \bar{f}_{t}\left(1-\bar{f}_{t}\right) d E .
$$

In this case, the traps and extended state occupation remain in equilibrium throughout the measurement, so there are no kinetic manifestations of the traps. This forms the basis for the quasistatic approximation to multiple trapping systems, which will be shown in detail in Sec. III, below.

With respect to the shallow levels, at $E_{t}>E_{F}$, we note in Eq. (22) and Fig. 2(b) that $\omega_{t}$ increases exponentially with energy, up to the maximum value

$$
\omega_{t m}=N_{c} \beta .
$$

Therefore, the relaxation of these levels, given by

$$
C_{\text {shallow }}^{*}(\omega)=\frac{q^{2}}{k_{B} T} \int_{E_{F}}^{E_{c}} g(E) \frac{\bar{f}_{t}\left(1-\bar{f}_{t}\right)}{1+i \omega / \omega_{t}(E)} d E,
$$

has to be calculated for the specific distribution of traps. In the following we calculate the two components of the frequency-dependent capacitance, for deep and shallow levels, in the specific case of an exponential distribution defined as

$$
g(E)=\frac{N_{t}}{k_{B} T_{0}} \exp \left[-\left(E_{c}-E\right) / k_{B} T_{0}\right] .
$$

Here, $N_{t}$ is the total volume density of localized states in the distribution, $k_{B}$ is Boltzmann's constant, and $T_{0}$ is a parameter with temperature unit that defines the depth of the distribution below $E_{c}$.

\section{E. Relaxation of an exponential distribution of traps: Deep levels}

For the deep levels we note that the following approximation is valid:

$$
\bar{f}_{t}\left(1-\bar{f}_{t}\right) \approx e^{\left(E_{t}-E_{F}\right) / k_{B} T} \quad\left(\text { at } \quad E_{t}>E_{F}\right) .
$$

Calculating the integral in Eq. (25) we obtain

$$
C_{\text {deep } 0}=\frac{q^{2} N_{t} e^{-\left(E_{c}-E_{F}\right) / k_{B} T_{0}}}{k_{B}\left(T+T_{0}\right)},
$$

and from Eqs. (21) and (26) we get the resistance dependence on Fermi level

$$
R_{\text {deep }}=\frac{k_{B}\left(T+T_{0}\right)}{\beta q^{2} N_{t} N_{c}} e^{\left(E_{c}-E_{F}\right)\left(1 / k_{B} T_{0}+1 / k_{B} T\right)} .
$$

\section{F. Relaxation of an exponential distribution of traps: Shallow levels}

For the shallow levels we find

$$
\bar{f}_{t}\left(1-\bar{f}_{t}\right) \approx e^{-\left(E_{t}-E_{F}\right) / k_{B} T} \quad\left(\text { at } \quad E_{t}>E_{F}\right) .
$$

Let us introduce the exponents

$$
a=1-\alpha=1-\frac{T}{T_{0}} .
$$

Equation (29) turns into

$$
C_{\text {shallow }}^{*}=\frac{q^{2} N_{t} e^{-E_{c} / k_{B} T_{0}+E_{F} / k_{B} T_{0}}}{k_{B}^{2} T T_{0}} \int_{E_{F}}^{E_{c}} \frac{e^{-a E / k_{B} T}}{1+i \omega \omega_{t m}^{-1} e^{-\left(E-E_{c}\right) / k_{B} T}} d E .
$$

In a previous work, ${ }^{36}$ by using a distribution with certain properties concerning the characteristic frequencies, it was shown that one can obtain the constant-phase element (CPE) behavior, with impedance $Z(\omega)=Q^{-1}(i \omega)^{-m}$. We treat the integral in Eq. (36) as described in Ref. 36. Using the change of variables $t=i \omega \omega_{t m}^{-1} e^{-\left(E-E_{c}\right) / k_{B} T}, t_{0}=i \omega / \omega_{t 0}$, and $t_{m}=i \omega / \omega_{t m}$, we obtain

$$
C_{\text {shallow }}^{*}=\frac{q^{2} N_{t} e^{-\left(E_{c}-E_{F}\right) / k_{B} T}}{k_{B} T_{0}}\left(\frac{i \omega}{\omega_{t m}}\right)^{-a}\left[\int_{t_{m}}^{t_{0}} \frac{t^{a-1}}{1-t} d t\right] .
$$

In the range of frequencies $\omega_{t 0} \ll \omega \ll \omega_{t m}$, we can set in the integral in Eq. (37) $t_{m} \approx 0$ and $t_{0} \approx \infty$. The final results are

$$
\begin{gathered}
C_{\text {shallow }}^{*}=Q_{\alpha}(i \omega)^{-a}, \\
Q_{\alpha}=\frac{q^{2} \pi N_{t}\left(N_{c} \beta\right)^{-a} e^{-\left(E_{c}-E_{F}\right) / k_{B} T}}{k_{B} T_{0} \sin (\pi a)} .
\end{gathered}
$$

Equation (38) represents a CPE element and dominates the capacitance $C_{\text {traps }}^{*}$ at $\omega_{t 0} \ll \omega \ll \omega_{t m}$. The reduction of the combination of the traps to a simple CPE model is indicated in Fig. 3. The CPE behavior of the impedance is, according to Eq. (38),

$$
Z_{\text {shallow }}=\frac{(i \omega)^{-\alpha}}{Q_{\alpha}} .
$$

By Laplace transform to the time domain, Eq. (40) is directly related to the classical results on dispersive (power-law) behavior of the mobility in transient experiments ${ }^{29}$ in disordered semiconductors. 

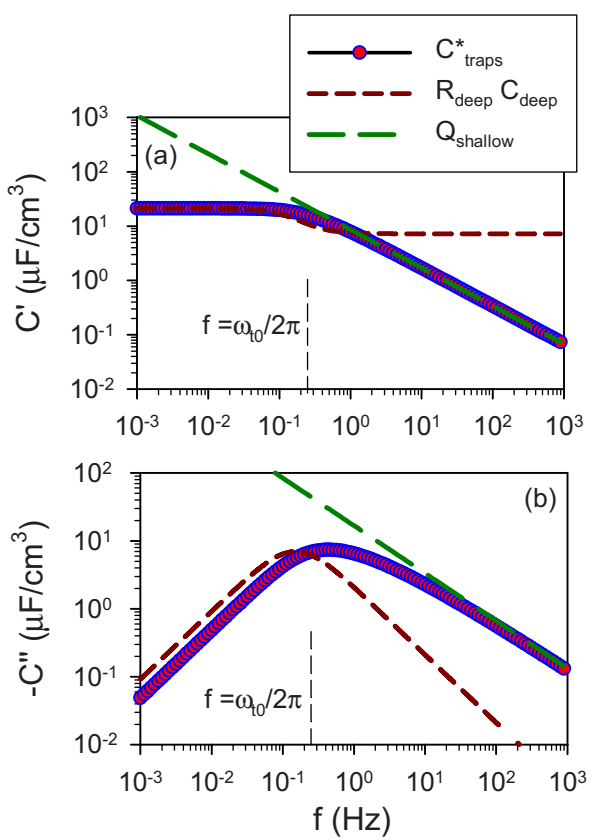

FIG. 4. (Color online) Representation of the real and imaginary parts of the frequency-dependent capacitance $C_{\text {traps }}^{*}$ for an exponential distribution of traps as a function of the frequency. Also shown are the approximations at low frequency $\left(C_{\text {deep }}^{*}\right)$, an $R C$ circuit, and high frequency $\left(C_{\text {shallow }}^{*}\right)$, a CPE element. The square point marks the deep trap frequency $\omega_{t 0}$. The parameters used in the calculation: $T=300 \mathrm{~K}, E_{F}=0.4 \mathrm{eV}, E_{c}=1 \mathrm{eV}, T_{0}=800 \mathrm{~K} \quad(\alpha=0.375), N_{c}$ $=10^{20} \mathrm{~cm}^{-3}, N_{t}=10^{20} \mathrm{~cm}^{-3}$, and $\beta=10^{-10} \mathrm{~m}^{2} \mathrm{~s}^{-1}$.

Figure 4 illustrates the behavior of the trap capacitance as a function of the frequency. In the representation, we denote $C^{\prime}=\operatorname{Re}\left(C^{*}\right)$ and $C^{\prime \prime}=\operatorname{Im} C^{*}$. It is observed that the series circuit of $R_{\text {deep }}$ and $C_{\text {deep } 0}$ is a good approximation to the exact capacitance in the low-frequency range $\left(\omega<\omega_{t 0}\right)$. It is also observed that the CPE in Eq. (38) is an excellent approximation to the exact result in the high-frequency range $\left(\omega_{t 0}\right.$ $\left.<\omega<\omega_{t m}\right)$.

Figure 5 shows the capacitance and impedance in the complex plane representation. (Note that the elementary trap resistances are connected in parallel, hence the macroscopic resistance of a film is inversely proportional to the volume of material.) At low frequencies (below $\omega_{t 0}$ ), the impedance behaves indeed as a series circuit of $R_{\text {deep }}$ and $C_{\text {deep } 0}$. In fact, it is observed in Fig. 5(a) that $R_{\text {deep }}$ and $C_{\text {deep } 0}$ provide a good approximation to the dc value $(\omega=0)$ of the impedance $R_{\text {traps }}(0)=\operatorname{Re}[Z(0)]$ and capacitance $C_{\text {traps }}=C_{\text {traps }}^{*}(0)$, respectively. These parameters are represented as a function of Fermi level in Fig. 6. Note that Eqs. (32) and (33) introduce a factor of error to the exact dc values of the exponential distribution. The reason for this is clear in Fig. 5(a): the values $R_{\text {deep }}$ and $C_{\text {deep0 }}$ provide the low-frequency arc in the capacitance plot, and therefore a contribution to the capacitance by the shallow levels, at higher frequency, is neglected. Nonetheless, Eqs. (32) and (33) constitute useful approximations with analytically closed expressions and describe well the Fermi-level dependence of $R_{\text {traps }}(0)$ and $C_{\text {traps }}$, as seen in Fig. 6.

Figure 7 shows in more detail the spectral features of the impedance. In Fig. 7, the time constant for electron capture
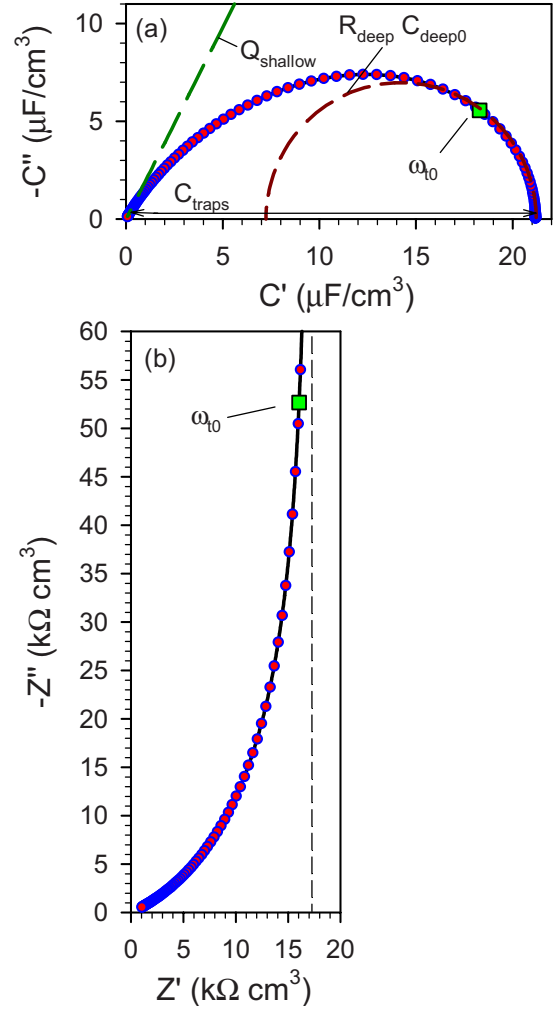

FIG. 5. (Color online) (a) Representation of the capacitance $C_{\text {traps }}^{*}$ and (b) correspondent impedance in the complex plot for an exponential distribution of traps. The square point marks the deep trap frequency $\omega_{t 0}$. In (a) are indicated in dashed lines the CPE approximation at high frequency (green line) and the series circuit, $R_{\text {deep }} C_{\text {deep } 0}$, at low frequency (brown line). The vertical line in (b) shows the value of low-frequency resistance. The parameters used in the calculation: $T=300 \mathrm{~K}, E_{F}=0.4 \mathrm{eV}, E_{c}=1 \mathrm{eV}, T_{0}=800 \mathrm{~K}$ $(\alpha=0.375), N_{c}=10^{20} \mathrm{~cm}^{-3}, N_{t}=10^{20} \mathrm{~cm}^{-3}$, and $\beta=10^{-10} \mathrm{~cm}^{2} \mathrm{~s}^{-1}$.

has been decreased with respect to Fig. 5, and we see that this modification has a large impact on the value of the trap resistance. The overall shape is similar to the simple two-trap model in Fig. 14(b), with a transition from the parallel resistance value observed at the intercept in Fig. 7(a) to the lowfrequency value in Fig. 7(c). The additional feature, introduced by the exponential distribution, is the CPE behavior, a tilted straight line of slope $\alpha$ in the intermediate frequency range, which is observed in Fig. 7(b).

\section{DEVICE MODELING}

Models for electronic (or ionic or mixed) devices are formulated with the appropriate transport and/or conservation equation for each kind of carrier. Models can vary widely depending on the number of material layers, different phases in each layer, and the boundary conditions that describe carrier transfer or blocking at the different interfaces. In Sec. II, we have formulated the trap model in the frequency domain using the standard approach that assumes the same average distribution of electronic levels at each point of the phase that shows such distribution. Therefore, whatever the device model, the presence of the exponential distribution implies 


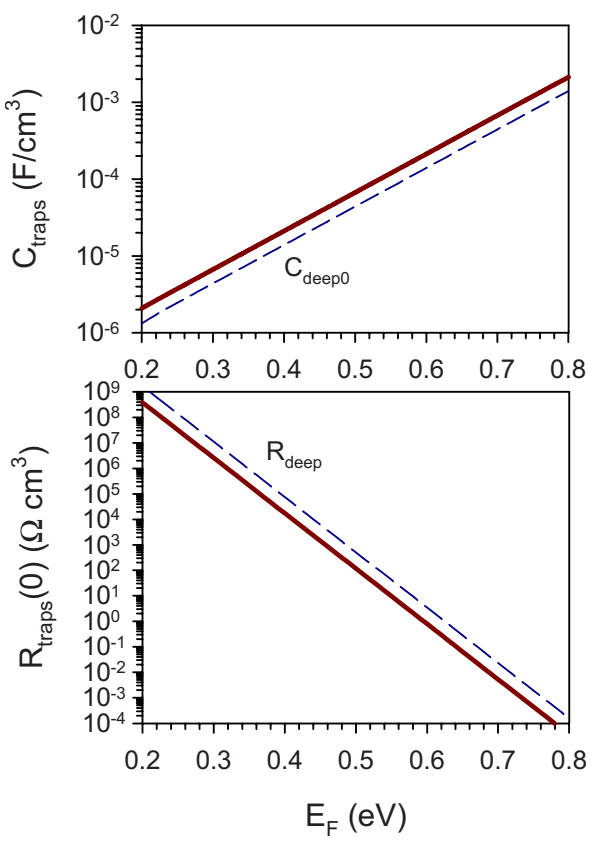

FIG. 6. (Color online) The thick red lines show the dc value of the (a) capacitance and (b) resistance for an exponential distribution of traps as a function of the Fermi level. In blue dashed lines are shown the dc values for the capacitance and resistance of the deep levels, according to the approximations discussed in the main text. The parameters used in the calculation: $T=300 \mathrm{~K}, E_{c}=1 \mathrm{eV}, T_{0}$ $=800 \mathrm{~K} \quad(\alpha=0.375), \quad N_{c}=10^{20} \mathrm{~cm}^{-3}, \quad N_{t}=10^{20} \mathrm{~cm}^{-3}$, and $\beta$ $=10^{-10} \mathrm{~cm}^{2} \mathrm{~s}^{-1}$.

that the development of Sec. II "hangs" at each point as part of the conservation equation, in addition to the specific transport features of the device model.
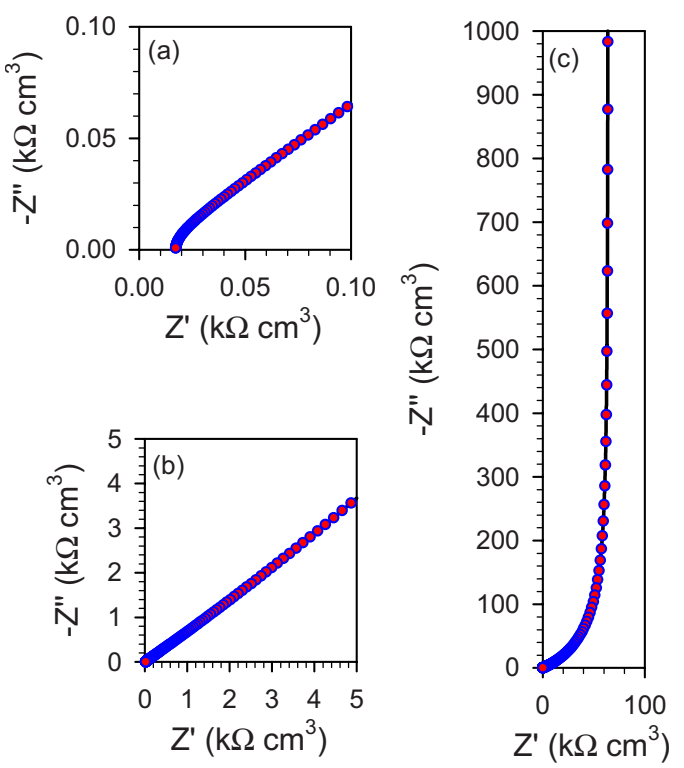

FIG. 7. (Color online) Representation of the impedance $Z_{\text {traps }}$ $=1 /\left(i \omega C_{\text {traps }}^{*}\right)$ for an exponential distribution of traps. (a) $-(\mathrm{c})$ show successive enlargement of the axis. The parameters used in the calculation: $T=300 \mathrm{~K}, \quad E_{F}=0.4 \mathrm{eV}, \quad E_{c}=1 \mathrm{eV}, \quad T_{0}=800 \mathrm{~K} \quad(\alpha$ $=0.375), N_{c}=10^{20} \mathrm{~cm}^{-3}, N_{t}=10^{20} \mathrm{~cm}^{-3}$, and $\beta=10^{-14} \mathrm{~cm}^{2} \mathrm{~s}^{-1}$. (a)

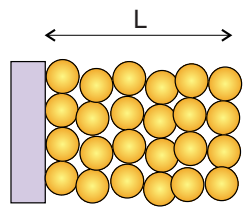

(b)

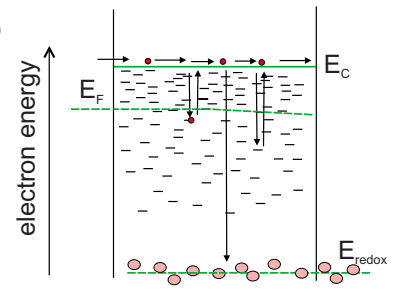

FIG. 8. (Color online) (a) Schematic representation of a porous semiconductor film, formed by connected nanoparticles deposited over a conducting substrate (shown at the left). (b) Electronic processes in the porous film, when it is immersed in a redox electrolyte. Electrons injected from the substrate diffuse by displacement in the extended states. They have a probability of being trapped and further released by localized states in the band gap. In addition, electrons in the conduction band have a chance to be captured by the oxidized form of the redox ions, which are indicted below around the redox potential level. The Fermi level is shown inclined, indicating a transient situation in which macroscopic diffusion of electrons occurs toward the right direction.

Obviously, device model can become quite complicated, especially in those cases in which the carrier distribution is heavily inhomogeneous. The trap occupation will vary from point to point, and this usually requires numerical solution. In order to illustrate the application of the trap model, we consider in this section the model of transport by diffusion coupled with trapping in a layer of thickness $L$. This model is not trivial and applies in a variety of situations, but it has the advantage that it is a one-dimensional problem in which steady-state carrier distribution is nearly homogeneous and can be solved analytically.

An example of application is presented in Fig. 8. The scheme shows a nanostructured and undoped metal-oxide semiconductor, deposited on a conducting substrate (left boundary), and immersed in electrolyte solution that compensates with ions any increase of electronic charge in the solid porous phase. ${ }^{9}$ Electrons can be injected and extracted in the film from the substrate, diffuse in the film, and are reflected at the outer edge (right boundary) of the semiconductor layer. This structure is of interest for a number of nanostructured electrochemical devices, ${ }^{9}$ among which the most prominent are the photoelectrochemical solar cells such as DSC. ${ }^{1,2}$ It is well documented that metal oxides such as $\mathrm{TiO}_{2}$ used in DSC exhibit an exponential distribution of electronic traps, ${ }^{18}$ and such distribution exerts an important influence over the electron diffusion coefficient. This subject has been reviewed recently ${ }^{25}$ using the quasistatic approximation, and in the following we will develop the complete dynamics of traps in the frequency domain.

The observation of trap dynamics in electron transport in nanostructured $\mathrm{TiO}_{2}$ has been reported in the time domain at very short-time scales, ${ }^{40,41}$ but to our knowledge, the ample body of experimental data in the frequency domain (see Ref. 
7 and references therein) has not been scanned in search of trap dynamics, very probably due to the lack of suitable models that expose the frequency response of the trap distribution. It is also likely that the dynamics of electronic traps is far too fast to be observed in the usual frequency range of impedance spectroscopy $(0.1 \mathrm{~Hz}-1 \mathrm{kHz})$. In the case of ionic solid conductors, such as electrochromic and Li battery materials, the trap dynamics is generally much slower and more likely to be clearly detected. So one important point in the following model is to justify the appearance of anomalous diffusion in the case of the exponential distribution of traps. Such model was developed using formal considerations, ${ }^{19}$ and it has been found very powerful for describing electrointercalation diffusion in solids. ${ }^{21,42}$ Recently, published impedance data ${ }^{43}$ seem to realize all the features of the diffusion-trap model, including anomalous diffusion features.

Another central aspect of photovoltaic applications, coupled with diffusion, is recombination, and this will also be considered below. In general, recombination means the loss of electronic carriers, by meeting another kind of carrier, as in electron-hole annihilation. In contrast to trapping process, in which the carriers are accumulated in certain localized states and can be retrieved, recombination is an irreversible process where the carriers are lost by transformation, and therefore, the free energy associated with such carriers does not contribute to energy output of the solar cell.

The general situation treated in this section is illustrated in Fig. 8. Injection (or extraction) of carriers causes diffusion along the extended states. While they advance, free carriers have the chance to be captured, and later released, by traps. In addition, free carriers have a possibility to be captured in recombination process. The recombination process specifically shown in Fig. 8 is the electron transfer from the metaloxide nanoparticles toward ionic species in solution (these are depicted around their own Fermi level, the redox potential). This is the dominant recombination mechanism in standard liquid electrolyte DSC, ${ }^{1}$ where the $I^{-} / I_{3}^{-}$redox couple is normally used to regenerate the oxidized dye molecules from the counterelectrode. The concentration of electron acceptor ions is very high and is not affected by charge transfer from the semiconductor. The exponential distribution of traps has been amply applied in the studies of DSC with a liquid redox electrolyte, and it is agreed that the lifetime dependence on Fermi level is well explained by the trapping effect; ${ }^{10,44,45}$ however, these studies all used the quasistatic approximation. We should also remark that DSC can be made also with organic electronic hole conductors filling the voids of the porous network, and the interfacial charge transfer implies electron-hole recombination. ${ }^{46}$

In Secs. III A-III C we derive first the theory of impedance for diffusion trapping in spatially restricted conditions and, thereafter, the model for recombination coupled with trapping. The main point is to reveal the effects of trap dynamics on diffusion and recombination phenomena beyond the quasistatic approach that was used in previous works. $^{24,25,45,47}$

\section{A. General features of the diffusion impedance}

Considering the different processes outlined in Fig. 8, we extend Eq. (4) to the transport-conservation equation as follows:

$$
\frac{\partial n_{c}}{\partial t}=-\frac{\partial n_{t}}{\partial t}-\frac{\partial J_{n}}{\partial x}-\frac{n_{c}}{\tau_{0}} .
$$

In Eq. (41) $\tau_{0}$ is the lifetime of electrons in the extended states and $J_{n}$ is the flux of free carriers at position $x$ that relates to the gradient of concentration by Fick's law,

$$
J_{n}=-D_{0} \frac{\partial n_{c}}{\partial x},
$$

where $D_{0}$ is the diffusion coefficient of the free electrons in extended states.

The analysis of diffusion impedance models has been presented in previous papers ${ }^{19,48,49}$ and we build on those results here. The solution of Eq. (41) for small ac perturbation, with a blocking boundary condition, is the general expression of the diffusion impedance in a film of thickness $L$,

$$
Z(s)=\left[\zeta_{m}(s) r_{m}\right]^{1 / 2} \operatorname{coth}\left\{L\left[\zeta_{m}(s) r_{m}\right]^{1 / 2}\right\} .
$$

Here $s=i \omega$ and $r_{m}$ is the transport impedance per unit length per area $(\Omega \mathrm{m})$,

$$
r_{m}=A R_{1}^{M} / L,
$$

where $R_{1}^{M}$ is the macroscopic transport resistance of the film of area $A . r_{m}$ is the reciprocal to the electronic conductivity $\sigma_{n}$

$$
r_{m}=\sigma_{n}^{-1}
$$

The conductivity relates to the free electron diffusion coefficient as

$$
\sigma_{n}=C_{0} D_{0}
$$

where $C_{0}$ is the specific chemical capacitance of the extended states ${ }^{48,50}$ defined in Eq. (13). Equation (46) is an expression of the Einstein relation. ${ }^{25}$ We note that the conductivity is given by

$$
\sigma_{n}=\frac{q^{2} n_{c} D_{0}}{k_{B} T},
$$

The element $\zeta_{m}$ in Eq. (43) adopts different forms, depending on the local processes included in the model (trapping, recombination, etc.). For the interpretation of the model, it is useful to represent Eq. (43) as a distributed equivalent circuit (a transmission line). The general impedance in Eq. (43) is represented in Fig. 9(a), and the transmission lines (b)-(f) represent specific models that will be discussed in this section. Note that Fig. 9(a) must be regarded as a continuous model in which the branching does not correspond to finite distances. The elements $r_{m}$, associated with diffusive transport, ${ }^{50}$ are distributed in the spatial direction and are continuously interrupted by transversal elements $\zeta_{m}$. This combination corresponds to the physical probabilities for electronic events in Fig. 8 and gives rise to the characteristic structure of a transmission line. Generally, linear equations for physical quantities varying in space with local dissipation are represented with transmission lines, for example, acoustic waves. ${ }^{51}$

We discuss first the simplest (ordinary) diffusion model, with no traps and no recombination, shown in Fig. 9(b). In 
(a)

general
diffusion

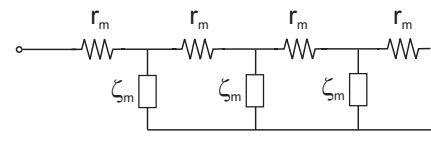

(b) ordinary

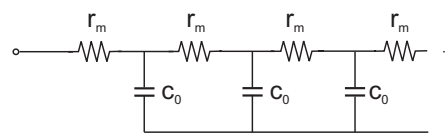

$r_{m} \quad r_{m} \quad r_{m} \quad r_{m}$

diffusion

and

recombination
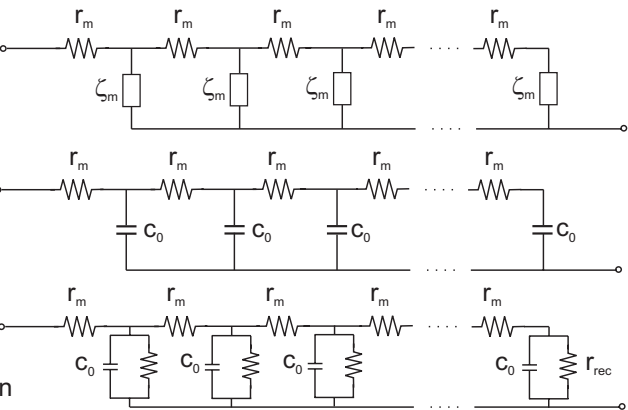

$r_{m}$

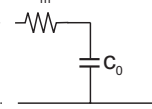

$r_{m}$ $-\mathrm{W}$

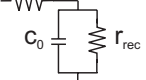

(d)
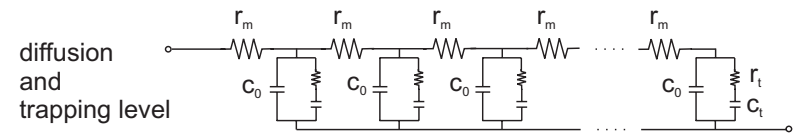

(e) diffusion

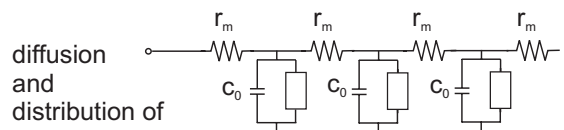

and

distribution of traps

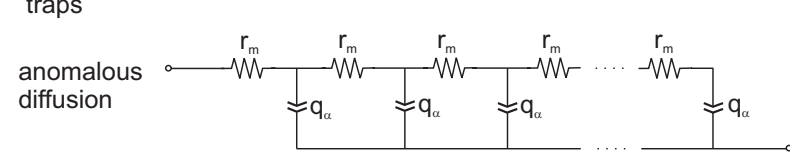

$r_{m}$

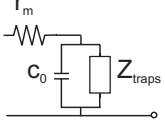

(f)

FIG. 9. (a) Transmission line representation of the diffusion impedance with the distributed diffusion resistance $r_{n}$ and the general transverse element $\zeta_{n}$. [(b)-(f)] Specific models are indicated, depending on the local electronic processes coupled with diffusion.

this model the distributed admittance $\zeta_{m}^{-1}$ consists on the chemical capacitance

$$
\zeta_{m}^{1}=C_{0} s=\left(C_{0}^{M} / A L\right) s .
$$

Here, $C_{0}^{M}$ is the macroscopic chemical capacitance of the film. Equation (43) gives

$$
Z(s)=R_{1}^{M}\left(\omega_{d 0} / s\right)^{1 / 2} \operatorname{coth}\left[\left(s / \omega_{d 0}\right)^{1 / 2}\right] .
$$

The characteristic transport frequency $\omega_{d 0}$ is the reciprocal of the transit time through the layer of thickness $L$,

$$
\omega_{d 0}=\frac{D_{0}}{L^{2}}=\frac{1}{R_{1}^{M} C_{0}^{M}} .
$$

The impedance spectrum of blocked diffusion is shown in Fig. 10(a). At high frequencies the spectrum exhibits the $45^{\circ}$ line (Warburg impedance) associated with diffusion. At frequencies lower than $\omega_{d 0}$ the spectrum is capacitive, and the low-frequency resistance is $R_{1}^{M} / 3$. This is an important feature, since the electronic conductivity of the semiconductor layer can be directly extracted from $R_{1}^{M}$, by Eqs. (44) and (45).

For diffusion and recombination, there appears a recombination resistance in parallel with the chemical capacitance in the transmission line [see Fig. 9(c) and Ref. 48]. This will be further discussed in Sec. III C. The transverse $\zeta$ impedance in this case is

$$
\zeta_{m}=\frac{r_{\mathrm{rec}}}{1+s / \omega_{\mathrm{rec}}} .
$$

The characteristic frequency of recombination is
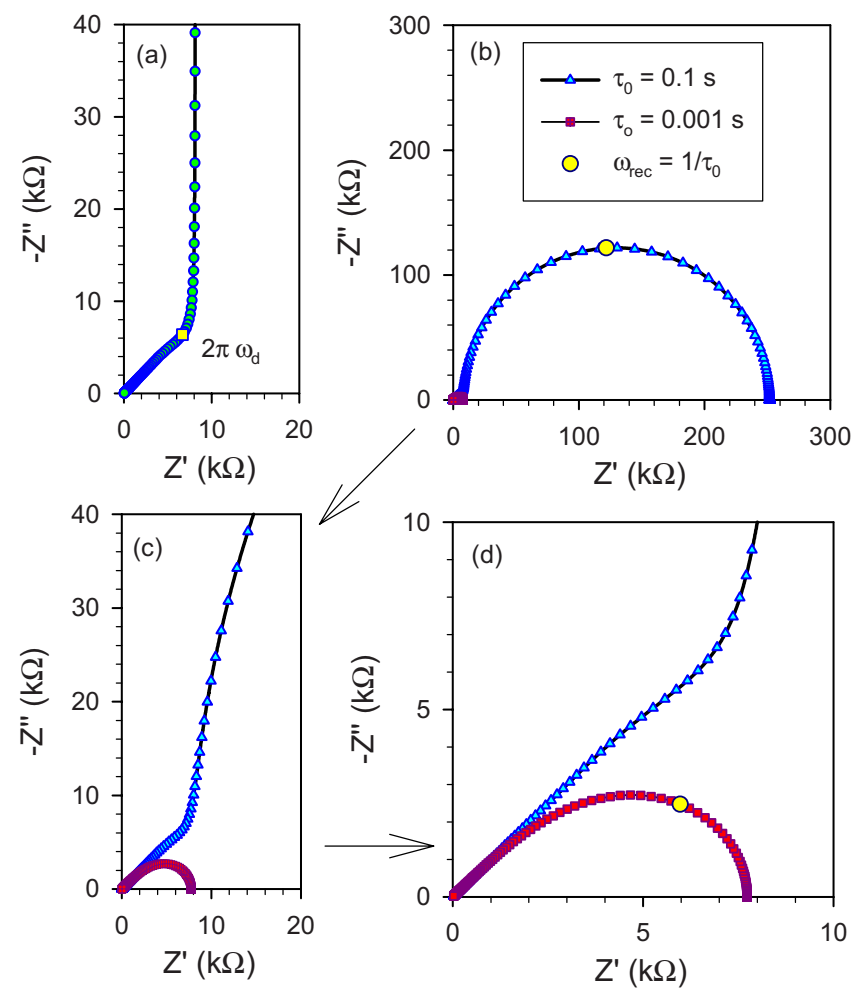

FIG. 10. (Color online) Representation of diffusionrecombination impedance of a film of thickness $L$. (a) Diffusion in the absence of recombination. The square point marks the angular frequency of turnover, related to $\omega_{d}=100 \mathrm{rad} \mathrm{s}^{-1}$. [(b),(c) and (d)] Diffusion-recombination impedance, showing two cases with different values of lifetime, as indicated. The yellow round point marks the effective lifetime frequency $\tau_{0}^{-1}$, with the values $\tau_{0}=10^{-1} \mathrm{~s}$ and $\tau_{0}=10^{-3} \mathrm{~s}$. The parameters used in the calculation: $T=300 \mathrm{~K}, E_{F}$ $=0.75 \mathrm{eV}, \quad E_{c}=1 \mathrm{eV}, \quad N_{c}=10^{18} \mathrm{~cm}^{-3}, \quad D_{0}=10^{-4} \mathrm{~cm}^{2} \mathrm{~s}^{-1}, \quad L$ $=10^{-3} \mathrm{~cm}$, and $A=1 \mathrm{~cm}^{2}$.

$$
\omega_{\text {rec } 0}=\tau_{0}^{-1},
$$

and the distributed recombination resistance is given by

$$
r_{\mathrm{rec}}=\frac{\tau_{0}}{C_{0}}=L A R_{3}^{M}
$$

where $R_{3}^{M}$ is the macroscopic recombination resistance of the layer. In contrast with Fig. 10(a), the spectra of diffusion recombination, shown in Figs. 10(b)-10(d), are resistive at low frequencies. There are two competing processes now, the transport across the layer $\left(\omega_{d 0}\right)$ and the carrier loss by recombination $\left(\omega_{\mathrm{rec} 0}\right)$. The shape of the spectra is regulated by the factor relating the characteristic frequencies, which can be expressed in several alternative ways, ${ }^{48}$

$$
\frac{\omega_{d 0}}{\omega_{\text {rec } 0}}=\frac{R_{3}^{M}}{R_{1}^{M}}=\left(\frac{L_{n}}{L}\right)^{2},
$$

where $L_{n}=\sqrt{D_{0} \tau_{0}}$ is the electron diffusion length. In the case of low recombination $\left(\omega_{\text {rec } 0} \ll \omega_{d 0}\right.$ or $\left.L_{n} \gg L\right)$, the carriers have plenty of time to cross the layer before they are lost. The spectrum is a recombination arc, shown in Fig. 10(b), and further discussed below. The diffusion Warburg is a 
small feature in the high-frequency part, clearly visible in the enlarged plot of Fig. 10(c), where we can see the start of the curvature of the capacitive part of Fig. 10(a), due to recombination. In the spectrum of Fig. 10(c) we can still determine the electronic conductivity from $R_{1}^{M} / 3$, i.e., the resistance at the turnover, and this method has been effectively applied in experiments. ${ }^{7,52}$

On the other hand, in the case of strong recombination $\left(\omega_{\mathrm{rec} 0} \gg \omega_{d 0}\right.$ or $\left.L_{n} \ll L\right)$ the injected carriers penetrate a restricted extent in the layer, and the boundary condition is irrelevant. The recombination resistance distorts the diffusion spectrum even at frequencies higher than the frequency of turnover $\omega_{d 0}$, and the spectrum adopts the form of the Gerischer impedance, shown more clearly in Fig. 10(d), which corresponds to diffusion recombination in semiinfinite space.

\section{B. Diffusion-trapping impedance}

The calculation of the impedance of diffusion and trapping is given in Ref. 49 for a single trap. The result is that the transverse element in Eq. (43) takes the form

$$
\zeta_{m}^{1}=\left[C_{0}+C_{\text {traps }}^{*}(s)\right] s .
$$

The resulting transmission line is shown in Fig. 9(d). According to Eq. (55), the $R C$ circuit of the trap is connected in parallel to the capacitance $C_{0}$ of the extended states.

Of interest here is the diffusion-trap impedance for an exponential distribution of localized states. It can be easily shown that the ac impedance, obtained using Eqs. (41) and (5), has the form of Eq. (55), where now, $C_{\text {traps }}^{*}$ is the full capacitance of the distribution [Eq. (18)], which we have characterized in Sec. II. The distributed equivalent circuit is shown in Fig. 9(e), where the transverse element should be regarded as that given in Fig. 3. So we can readily calculate the shape of the spectra by including Eq. (55) in Eq. (43).

A first set of results is shown in Fig. 11. In this example, the lowest trap frequency $\omega_{t 0}$ is larger than the frequency of transit time $\omega_{d n}$. Therefore, the overall shape of the impedance spectra, shown in Fig. 11(a), is the same as that of diffusion without traps, shown in Fig. 10(a). The kinetic effect of trapping appears as a modification of the Warburg domain, at high frequencies, shown in Figs. 11(b) and 11(c). We find two interesting features in this high-frequency range. First, at the highest frequencies, Fig. 11(c), we observe the Gerischer impedance, associated with diffusion recombination, and shown before in Fig. 10(d). This, in fact, occurs at frequencies higher than $\omega_{t m}$, which is for the fastest trap, i.e., the upper cutoff frequency of the distribution. The appearance of the Gerischer impedance is due to the fact that above $\omega_{t m}$, the trap capacitors are shunted, hence the trap behaves like a pure resistance, and the circuit in Fig. 9(d) reduces to that in Fig. 9(c).

In the intermediate frequency range $\left(\omega<\omega_{t m}\right)$, we observe in Fig. 11(c) a diffusion line with slope lower than $45^{\circ}$. This indicates the occurrence of anomalous diffusion, ${ }^{19}$ and this is caused by the reduction of $Z_{\text {traps }}$ to a CPE, as pointed out in Fig. 3 and discussed in Sec. II F. The CPE replaces the pure capacitance $C_{0}$ of ordinary diffusion, and the transmission
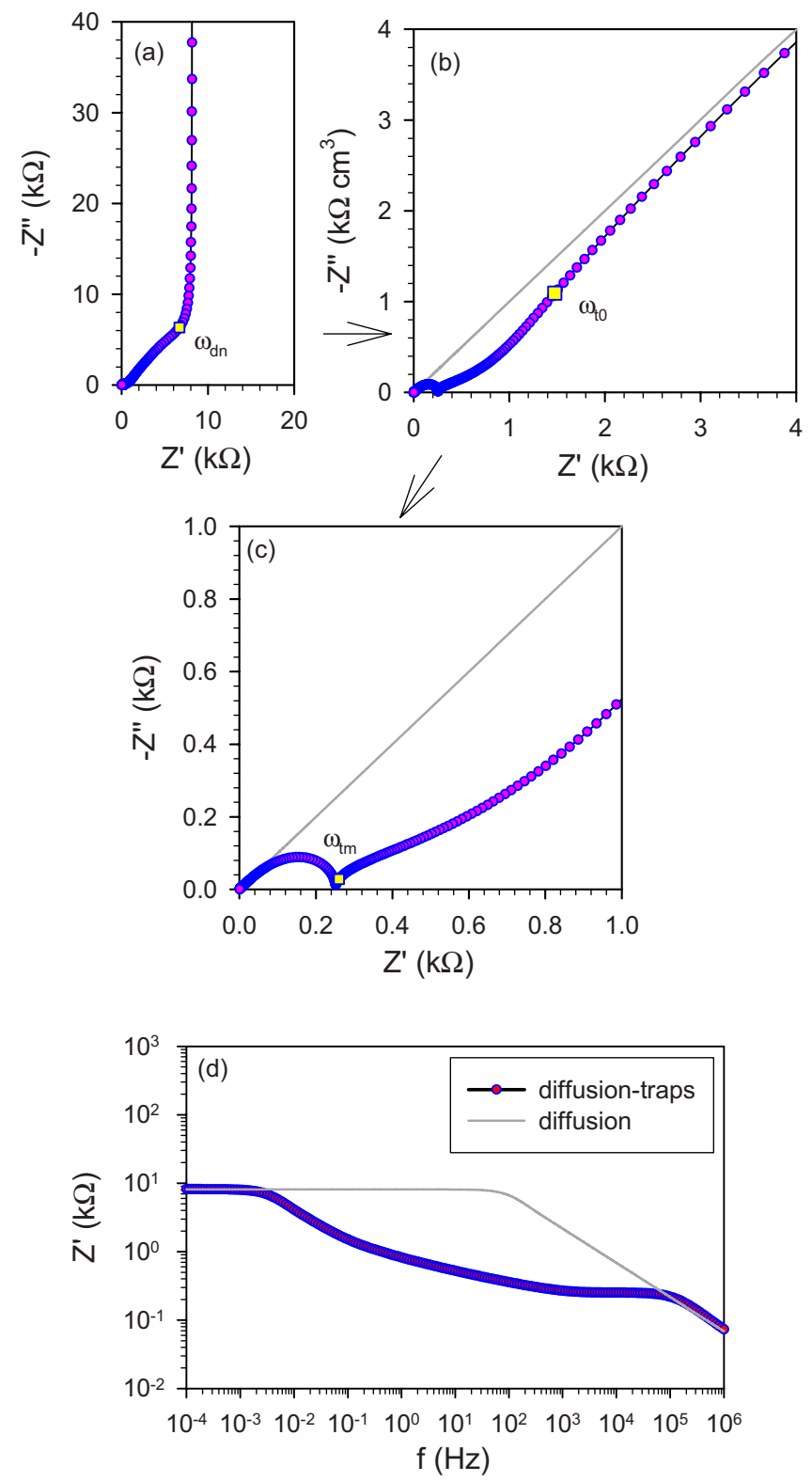

FIG. 11. (Color online) Representation of the impedance of diffusion in a film of thickness $L$, with an exponential distribution of traps. [(a)-(c)] Impedance spectra in the complex plane. The gray thin line is the impedance of diffusion without traps. The yellow square points mark the diffusion frequency $\omega_{d n}=\left(C_{0} / C_{\text {traps }}\right) \omega_{d 0}$, the cutoff frequency $\omega_{t m}$, and the deep traps frequency $\omega_{t 0}$. $(\mathrm{d})$ The real part of the impedance as a function of the frequency. The parameters used in the calculation: $T=300 \mathrm{~K}, E_{F}=0.75 \mathrm{eV}, E_{c}=1 \mathrm{eV}$, $T_{0}=1000 \mathrm{~K} \quad(\alpha=0.33), \quad N_{c}=10^{18} \mathrm{~cm}^{-3}, \quad N_{t}=10^{20} \mathrm{~cm}^{-3}, \quad D_{0}$ $=10^{-4} \mathrm{~cm}^{2} \mathrm{~s}^{-1}, L=10^{-3} \mathrm{~cm}$, and $A=1 \mathrm{~cm}^{2}$.

line of Fig. 9(e) adopts, in a restricted frequency range, the form of Fig. 9(f), which is the anomalous diffusion model. ${ }^{19,53}$

Let us return to the global spectrum shown in Fig. 10(a). Although it has the form of ordinary diffusion, as already commented, there is a strong change of the characteristic frequency of turnover, $\omega_{d n}$, with respect to that of free diffusion, $\omega_{d 0}$. This is clearly appreciated in the representation of the impedance as a function of the frequency [Fig. 11(d)], 
where both models are shown, for diffusion with and without traps. Here it is seen that the frequency of turnover is lowered by five decades of frequency. The reason for this is that, even though the trap relaxation occurs only at high frequencies, the traps strongly increase the equilibrium capacitance in the low-frequency range, which is given by $C_{\text {traps }}[\mathrm{Eq}$. (27)], as mentioned in Sec. II D. Therefore, the turnover frequency from the Warburg to the capacitive part has the value

$$
\omega_{d n}=\frac{D_{n}}{L^{2}}=\frac{1}{R_{1}\left(C_{0}^{M}+C_{\text {traps }}^{M}\right)} .
$$

Comparing with Eq. (50), we observe that the measured diffusion coefficient in the presence of traps is

$$
D_{n}=\frac{C_{0}}{C_{0}+C_{\text {traps }}} D_{0} .
$$

In general, $D_{n}$ can be interpreted as a chemical diffusion coefficient. $^{25,47}$ Note that the measured conductivity (from $R_{1}$ ) is not modified, since $\sigma_{n}$ is the same as that in Eq. (46),

$$
\sigma_{n}=C_{n} D_{n}=\left(C_{0}+C_{\text {traps }}\right) D_{n}=C_{0} D_{0} .
$$

Equation (58) corresponds to the generalized Einstein relation. $^{25}$

These results are an application of the quasistatic approximation $^{24}$ that was mentioned in Sec. I. If the trapping dynamics is fast, then the system can be described in terms of the transport model of the trap-free system. There is a modification of the measured time constant, i.e., the chemical diffusion coefficient, which is reduced by the additional capacitance of the traps. However, the dc quantities, such as the dc conductivity, are not modified at all. ${ }^{25}$

In Fig. 12 we plot the impedance of diffusion trapping with another set of parameters and we obtain a very different result. The change is due to the fact that trapping kinetics, in this case, are slower than the transit time. Thus, the shape of spectra [Fig. 12(a)] is not that of diffusion, but instead, we obtain the impedance response of the exponential distribution of traps, shown in Figs. 5 and 7. As before, at high frequency we obtain the Gerischer impedance, which is the manifestation of diffusion trapping. In Fig. 12(c) we observe that the low-frequency resistance is mainly due to traps in this case and not associated to the transport resistance. Therefore, the quasistatic approximation is invalid in this case, and we cannot infer the conductivity from the lowfrequency resistance, in contrast with the previous example.

\section{Trapping-recombination impedance}

From previous general analysis of the impedance of solar cells, we know that recombination introduces a resistance in parallel to the chemical capacitance. ${ }^{54}$ This has been already remarked above in Fig. 10(b). In the case in which the diffusion transit time, $L^{2} / D_{0}$, is much faster than the electron lifetime $\tau_{0}$, the impedance response is basically given by a recombination arc. In the following, we wish to calculate the modifications of the recombination impedance by the effect of the exponential distribution of traps. The result we expect is a resistance in parallel with the full capacitance $C^{*}(\omega)$ of
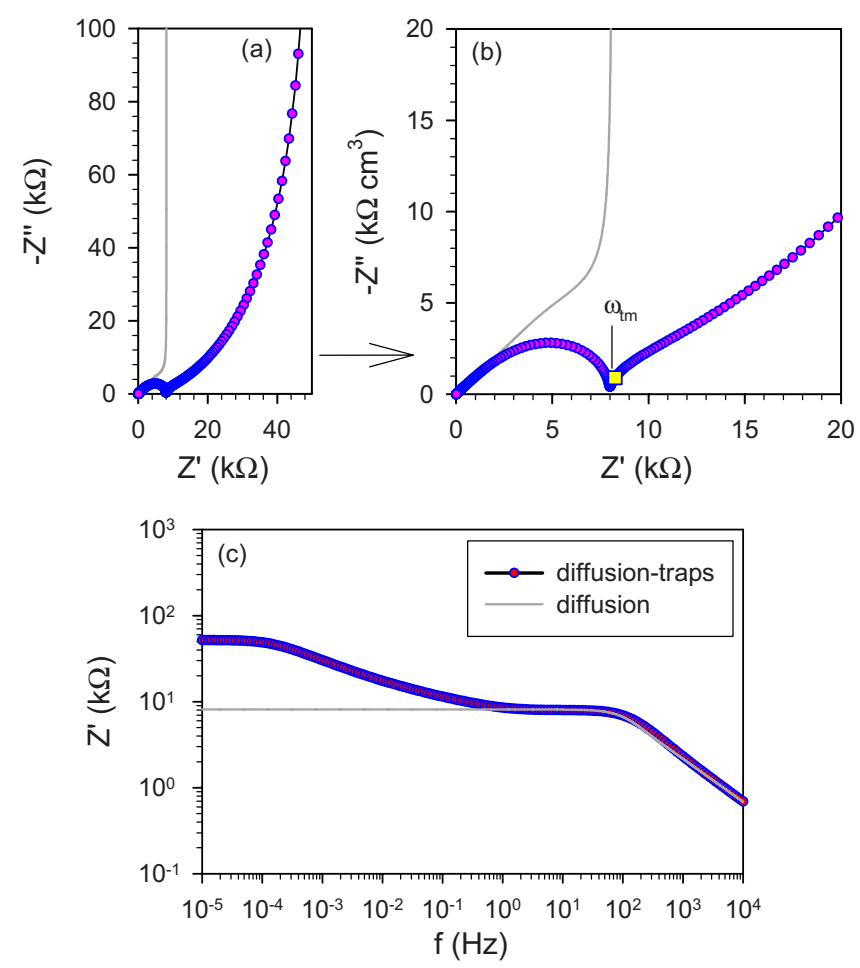

FIG. 12. (Color online) Representation of the impedance of diffusion in a film of thickness $L$, with an exponential distribution of traps. (a) and (b) show the impedance in the complex plane. The gray thin line is the impedance of diffusion without traps. The yellow square point marks the cutoff frequency $\omega_{t m}$. (c) shows the real part of the impedance as a function of the frequency. The parameters used in the calculation: $T=300 \mathrm{~K}, E_{F}=0.75 \mathrm{eV}, E_{c}=1 \mathrm{eV}$, $T_{0}=1000 \mathrm{~K} \quad(\alpha=0.33), \quad N_{c}=10^{18} \mathrm{~cm}^{-3}, \quad N_{t}=10^{20} \mathrm{~cm}^{-3}, \quad D_{0}$ $=10^{-4} \mathrm{~cm}^{2} \mathrm{~s}^{-1}, L=10^{-3} \mathrm{~cm}$, and $A=1 \mathrm{~cm}^{2}$.

the DOS, related to both the extended states and exponential distribution.

In order to show this, we consider that diffusive transport is very fast in the system of Fig. 8. This implies that carrier densities are homogeneous. Integrating Eq. (41) between the extracting boundary at $x=0$ and the blocking boundary at $x$ $=L$, we obtain

$$
\frac{\partial n_{c}}{\partial t}=-\frac{\partial n_{t}}{\partial t}-\frac{n_{c}}{\tau_{0}}+\frac{J_{n 0}}{L} .
$$

For a small perturbation in the frequency domain, Eq. (59) gives

$$
i \omega\left(\hat{n}_{c}+\hat{n}_{t}\right)+\frac{\hat{n}_{c}}{\tau_{0}}=\frac{\hat{J}_{n 0}}{L} .
$$

Using Eq. (20), we can write Eq. (60) as

$$
\left\{i \omega\left[C_{0}+C_{\text {traps }}^{*}(\omega)\right]+\frac{1}{r_{\text {rec }}}\right\} \hat{V}=\frac{\hat{J}_{n 0}}{L},
$$

where the recombination resistance is defined in Eq. (51).

The impedance of the film, per unit area, is 


$$
Z=\frac{\hat{V}}{q \hat{J}_{n 0}}=\frac{1}{L} \frac{r_{\mathrm{rec}}}{1+i \omega r_{\mathrm{rec}}\left[C_{0}+C_{\mathrm{traps}}^{*}(\omega)\right]} .
$$

This shows, as anticipated, that recombination introduces the resistance $r_{\text {rec }}$ in parallel to the total frequency-dependent capacitance $C^{*}(\omega)$ of Eq. (17).

Let us assume that the frequencies of the measurement are lower than the frequency $\omega_{t 0}$. In this case Eq. (20) reduces to

$$
\hat{n}_{t}=\frac{C_{\text {traps }}}{C_{0}} \hat{n}_{c},
$$

where $C_{\text {traps }}$ is given in Eq. (27). Equation (63) is the rigorous formulation of the quasistatic approximation ${ }^{24}$ for small modulation quantities. It means that the modulation of the trapped charge follows instantaneously the modulation of the free charge, as mentioned before, and the ratio of these quantities is given by equilibrium chemical capacitances of the corresponding states. If Eq. (63) holds, then from Eq. (62) we arrive at the impedance

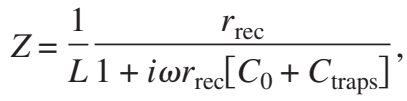

and this can be written

$$
Z=\frac{1}{L} \frac{r_{\text {rec }}}{1+i \omega \tau_{n}},
$$

where, by Eq. (53),

$$
\tau_{n}=\frac{C_{0}+C_{\text {traps }}}{C_{0}} \tau_{0}
$$

Equation (64) means that if $\omega \ll \omega_{t 0}$ the relaxation dynamics is the same as that of free electrons. ${ }^{54}$ There are no kinetic effects of traps. The traps, however, modify the time constant for recombination by the ratio of equilibrium capacitances [Eq. (66)]. This is because displacing the Fermi level requires to discharge the traps, as was first noted by Rose. ${ }^{22,23}$ The quasistatic approximation considerably simplifies the analysis of experimental techniques that probe the properties of DSC; see, for example, Refs. 10 and 45 that discuss the experimental determination of Eq. (66).

In Fig. 13 we have plotted the impedance of the exponential distribution of traps with the parallel recombination resistance [Eq. (62)]. We have neglected the free charge capacitance $C_{0}$ since this is usually not significant in comparison to $C_{\text {traps, }}^{*}$ unless $E_{F}$ is very close to the conduction-band edge. Now there are two characteristic frequencies in the system: $\omega_{t 0}$ indicates the onset of trap relaxation and CPE behavior, as discussed above, and $\omega_{\mathrm{rec}}=\tau_{n}^{-1}$ is the effective lifetime frequency.

In Fig. 13(a) we have $\omega_{t 0} \gg \omega_{\text {rec }}$, therefore the traps relaxation discussed in Fig. 7, occurs undisturbed at very high frequencies. When lowering the frequency, the impedance closes toward the real axis by the effect of recombination resistance, as in Fig. 10(b). The recombination $\operatorname{arc}^{54}$ has the characteristic frequency $\tau_{n}^{-1}$.

In Fig. 13(b) we have still $\omega_{t 0}>\omega_{\text {rec }}$, but their values are closer than in the previous example, and in this case the trap
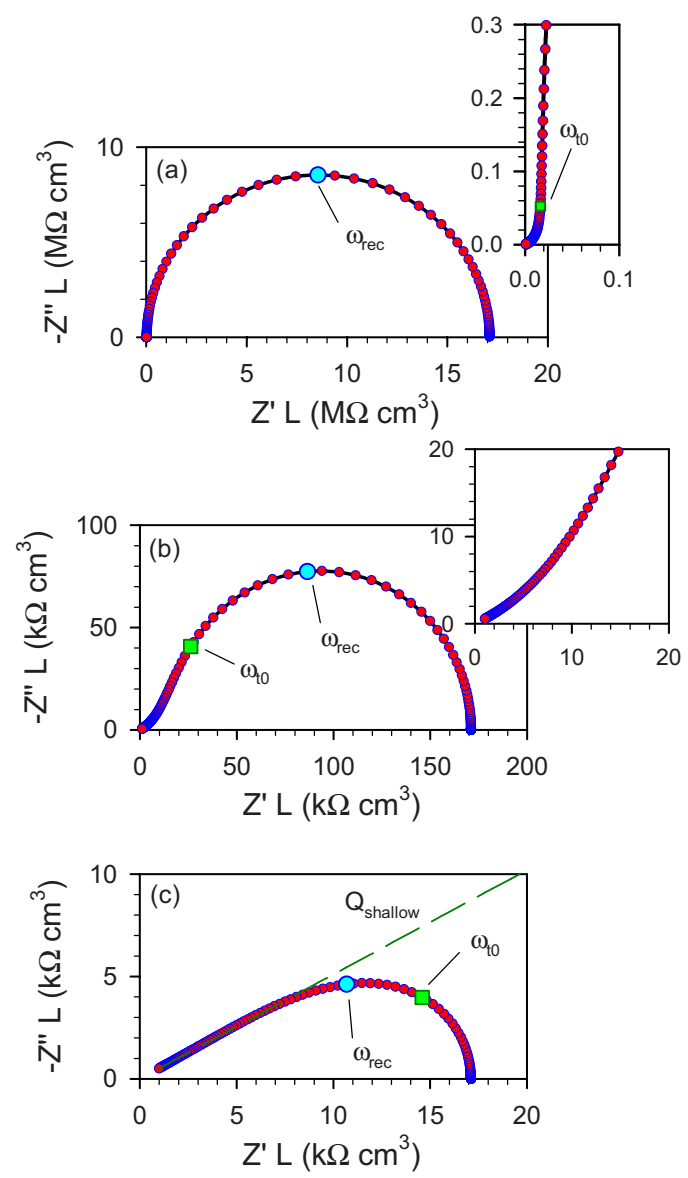

FIG. 13. (Color online) Repesentation of impedance of a film of thickness $L$, with an exponential distribution of traps with recombination from the conduction band. The green square point marks the deep trap frequency $\omega_{t 0}$, and the blue round point marks the effective lifetime frequency $\tau_{n}^{-1}$, with the values (a) $\tau_{0}=1 \mathrm{~s}$ and $\tau_{n}$ $=362 \mathrm{~s}$, (b) $\tau_{0}=0.01 \mathrm{~s}$ and $\tau_{n}=3.62 \mathrm{~s}$, and (c) $\tau_{0}=0.001 \mathrm{~s}$ and $\tau_{n}=0.362 \mathrm{~s}$. In (c) in green dashed line the CPE approximation at high frequency is indicated. The parameters used in the calculation: $T=300 \mathrm{~K}, E_{F}=0.4 \mathrm{eV}, E_{c}=1 \mathrm{eV}, T_{0}=800 \mathrm{~K} \quad(\alpha=0.375), N_{c}$ $=10^{20} \mathrm{~cm}^{-3}, N_{t}=10^{20} \mathrm{~cm}^{-3}$, and $\beta=10^{-10} \mathrm{~cm}^{2} \mathrm{~s}^{-1}$.

relaxation is visible as a modification of the recombination arc in the high-frequency part. Finally, in Fig. 13(c), we have $\omega_{t 0}<\omega_{\text {rec }}$. In this case the recombination resistance is smaller than the trap resistance. Therefore, the CPE behavior of trap relaxation is fully visible and is converted into an arc at the lower frequencies by the parallel recombination resistance.

It should be recalled that Fig. 13 represents a homogeneous model of recombination, in which carrier transport is infinitely fast, as assumed above. The impedance patterns of Fig. 13 are remarkably similar to those of the diffusionrecombination impedance in Figs. 10(b) and 10(c). In principle, this similarity introduces an ambiguity of models that could lead to mistake interpretation of trapping recombination (or trapping reaction) as diffusion-recombination results. However, from the Fermi-level dependence of the resistance in the high-frequency straight part, both models, diffusion and traps, should be readily distinguished. Indeed, in the diffusion model the high-frequency feature gives the transport 
resistance $R_{1}$, which depends on the potential as $\exp \left[\left(E_{c}\right.\right.$ $\left.-E_{F}\right) / k_{B} T$ ] [Eq. (47)]. In contrast, the Fermi-level dependence of the traps resistance is much more steep, as indicated in Eq. (33) and in Fig. 6(b). Therefore, we should emphasize that the spectra should be monitored over a sufficient variation of bias potential in order to determine the correct interpretation. In experimental results of impedance of DSC, the mentioned transport resistance dependence of Fermi level was observed, ${ }^{7,55}$ it therefore appears that trap relaxation is quite fast in nanostructured $\mathrm{TiO}_{2}$ surrounded with liquid electrolyte, and the widespread application of the quasistatic approximation is well justified.

The extension of the approach presented in this section, for nonhomogeneous device models, requires a twofold numerical solution. First, steady-state equations must be solved to find the local values of charge density at each point of the device. Then, such information allows us to write the transport equations for small ac quantities, which contain also dc parameters. An example is given in Ref. 50.

\section{CONCLUSIONS}

The relaxation of an exponential distribution of traps in the frequency domain contains two main features. At low frequency the distribution gives the normal relaxation of a single trap, i.e., a $R C$ series circuit. At high frequency the distribution responds as a constant-phase element. The CPE exponent is independent of the Fermi-level position but changes with the temperature as $\alpha=T / T_{0}$. The transition between the two features occurs roughly at the frequency of the highest occupied trap level at the position of the Fermi level. In the presence of diffusion and recombination, the interplay between the frequency of the highest occupied trap level and the effective (chemical) diffusion coefficient or effective lifetime determines the shape of impedance spectra. In the presence of very slow traps, the quasistatic approximation is not valid, since the low-frequency response is governed by the trap kinetics. We have found similarities between diffusionrecombination and trapping-recombination impedance models. Therefore, a careful analysis of experimental data is necessary, regarding these models, and exploring a wide window of potential and if possible temperature seems recommended to identify correctly the trap relaxation by impedance spectroscopy. The variation of impedance parameters with bias potential and temperature in agreement with the model should provide strong evidence for the identification of the trap kinetics.

\section{ACKNOWLEDGMENTS}

The work was supported by MEC under Project No. MAT2007-62982, HOPE Project No. under Grant No. CSD2007-00007 (Consolider-Ingenio 2010), and Generalitat Valenciana under Project No. ACOMP07/103. I am grateful to Henk Bolink for discussions on organic conductors.

\section{APPENDIX: IMPEDANCE AND CAPACITANCE OF SIMPLE COMBINATIONS OF TRAPS}

The frequency-dependent capacitance of a single trap is

$$
C_{t 1}^{*}(\omega)=\frac{C_{t 1}}{1+i \omega / \omega_{t 1}} .
$$
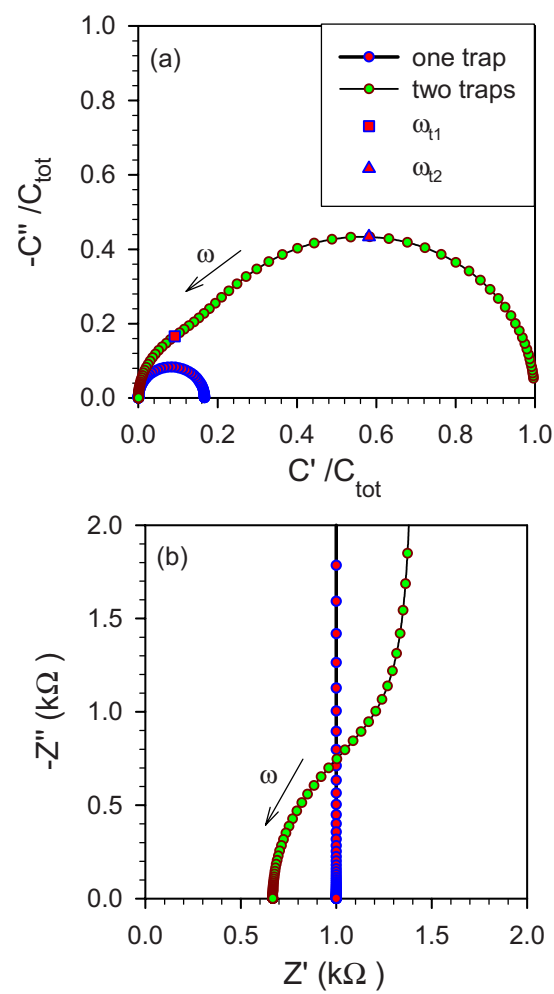

FIG. 14. (Color online) Representation of (a) capacitance and (b) impedance in the complex plane, for single and double trap models, as indicated. Parameters: $C_{t 1}=10^{-3} \mathrm{~F}, R_{t 1}=10^{3} \Omega, \omega_{t 1}$ $=1 \mathrm{rad} \mathrm{s}^{-1}, C_{t 1}=5 \times 10^{-3} \mathrm{~F}, R_{t 1}=2 \times 10^{3} \Omega, \omega_{t 1}=0.1 \mathrm{rad} \mathrm{s}^{-1}$, and $C_{\text {tot }}=C_{t 1}+C_{t 2}$.

Here, $C_{t 1}$ is the trap capacitance and $\omega_{t 1}$ is a constant, the characteristic trap frequency. The impedance associated with Eq. (A1) is

$$
Z_{t 1}(\omega)=R_{t 1}+\frac{1}{i \omega C_{t 1}}
$$

where the trap resistance is defined as

$$
R_{t 1}=\frac{1}{\omega_{t 1} C_{t 1}} .
$$

From Eq. (A2) we see that the equivalent circuit of the trap is $R C$ series. In the complex plane the capacitance forms an arc, of width $C_{t 1}$, while impedance forms a vertical line, being the resistance constant at all frequencies. This is illustrated in Fig. 14.

When we combine two traps, the $R C$ circuits are in parallel, as indicated in Fig. 1, so we have

$$
C_{\mathrm{tot}}^{*}(\omega)=\frac{C_{t 1}}{1+i \omega / \omega_{t 1}}+\frac{C_{t 2}}{1+i \omega / \omega_{t 2}} .
$$

This forms a double arc in the complex capacitance plane, as shown in Fig. 14(a). If we calculate the real part of the impedance, $Z_{\mathrm{tot}}=1 / i \omega C_{\mathrm{tot}}^{*}$, we obtain 


$$
\operatorname{Re}\left[Z_{\mathrm{tot}}(\omega)\right]=\frac{R_{t 1} R_{t 2}\left[R_{t 1} \omega_{t 1}^{2}+R_{t 2} \omega_{t 2}^{2}+\left(R_{t 1}+R_{t 2}\right) \omega^{2}\right]}{\left(R_{t 1} \omega_{t 1}+R_{t 2} \omega_{t 2}\right)^{2}+\left(R_{t 1}+R_{t 2}\right)^{2} \omega^{2}} .
$$

In the high-frequency limit this gives

$$
\operatorname{Re}\left[Z_{\mathrm{tot}}(\omega \rightarrow \infty)\right]=\frac{R_{t 1} R_{t 2}}{R_{t 1}+R_{t 2}} .
$$

Therefore, the impedance intersects the $x$ axis at the value of the parallel combination of the trap resistances. This is because the impedances of the capacitors vanish at high frequency. On the other hand, the low-frequency limit of the real part of the impedance depends on the capacitances (via the characteristic frequencies),

$$
\operatorname{Re}\left[Z_{\mathrm{tot}}(0)\right]=R_{t 1} R_{t 2} \frac{R_{t 1} \omega_{t 1}^{2}+R_{t 2} \omega_{t 2}^{2}}{\left(R_{t 1} \omega_{t 1}+R_{t 2} \omega_{t 2}\right)^{2}} .
$$

The impedance rises vertically when the frequency decreases, but it makes a transition between the values in Eqs. (A6) and (A7) [see Fig. 14(b)]. *bisquert@fca.uji.es

${ }^{1}$ B. O’ Regan and M. Grätzel, Nature (London) 353, 737 (1991).

${ }^{2}$ J. Bisquert, D. Cahen, S. Rühle, G. Hodes, and A. Zaban, J. Phys. Chem. B 108, 8106 (2004).

${ }^{3}$ C. J. Brabec, N. S. Sariciftci, and J. C. Hummelen, Adv. Math. 11, 15 (2001).

${ }^{4}$ P. Peumans, U. Uchida, and S. R. Forrest, Nature (London) 425, 158 (2003)

${ }^{5}$ J. H. Burroughes, D. D. C. Bradley, A. R. Brown, R. N. Marks, K. MacKay, R. H. Friend, P. L. Burn, and A. B. Holmes, Nature (London) 347, 539 (1990).

${ }^{6}$ S. R. Forrest, Nature (London) 428, 911 (2004).

${ }^{7}$ Q. Wang, S. Ito, M. Grätzel, F. Fabregat-Santiago, I. Mora-Seró, J. Bisquert, T. Bosshoa, and H. Imai, J. Phys. Chem. B 110, 19406 (2006)

${ }^{8}$ B. Ramachandhran, H. G. A. Huizing, and R. Coehoorn, Phys. Rev. B 73, 233306 (2006).

${ }^{9}$ J. Bisquert, Phys. Chem. Chem. Phys. 10, 49 (2008).

${ }^{10}$ L. M. Peter, J. Phys. Chem. C 111, 6601 (2007).

${ }^{11}$ M. C. J. M. Vissenberg and M. Matters, Phys. Rev. B 57, 12964 (1998).

${ }^{12}$ P. Servati, A. Nathan, and G. A. J. Amaratunga, Phys. Rev. B 74, 245210 (2006).

${ }^{13}$ G. Paasch and S. Scheinert, J. Appl. Phys. 101, 024514 (2007).

${ }^{14}$ M. M. Mandoc, B. de Boer, G. Paasch, and P. W. M. Blom, Phys. Rev. B 75, 193202 (2007).

${ }^{15}$ Z. Chiguvare and V. Dyakonov, Phys. Rev. B 70, 235207 (2004).

${ }^{16}$ M. D. Levi and D. Aurbach, J. Phys. Chem. B 109, 2763 (2005).

${ }^{17}$ J. Bisquert, Phys. Chem. Chem. Phys. 5, 5360 (2003).

${ }^{18}$ J. Bisquert, F. Fabregat-Santiago, I. Mora-Seró, G. GarciaBelmonte, E. M. Barea, and E. Palomares, Inorg. Chim. Acta 361, 684 (2008).

${ }^{19}$ J. Bisquert and A. Compte, J. Electroanal. Chem. 499, 112 (2001).

${ }^{20}$ J.-W. Lee and S.-I. Pyun, Electrochim. Acta 50, 1777 (2005).

${ }^{21}$ C. Montella, R. Michel, and J. P. Diard, J. Electroanal. Chem. 608, 37 (2007).

${ }^{22}$ A. Rose, RAC Rev. 12, 362 (1951).

${ }^{23}$ A. Rose, Concepts in Photoconductivity and Allied Problems (Interscience, New York, 1963).

${ }^{24}$ J. Bisquert and V. S. Vikhrenko, J. Phys. Chem. B 108, 2313 (2004).

${ }^{25}$ J. Bisquert, Phys. Chem. Chem. Phys. (to be published 2008).
${ }^{26}$ L. M. Peter, A. B. Walker, G. Boschloo, and A. Hagfeldt, J. Phys. Chem. B 110, 13694 (2006).

${ }^{27}$ M. E. Gershenson, V. Podzorov, and A. F. Morpurgo, Rev. Mod. Phys. 78, 973 (2006).

${ }^{28}$ J. Orenstein and M. Kastner, Phys. Rev. Lett. 46, 1421 (1981).

${ }^{29}$ T. Tiedje and A. Rose, Solid State Commun. 37, 49 (1981).

${ }^{30}$ A. L. Roest, P. E. de Jongh, and D. Vanmaekelbergh, Phys. Rev. B 62, 16926 (2000).

${ }^{31}$ D. L. Loose, J. Appl. Phys. 46, 2204 (1975).

${ }^{32}$ F. Fabregat-Santiago, I. Mora-Seró, G. Garcia-Belmonte, and J. Bisquert, J. Phys. Chem. B 107, 758 (2003).

${ }^{33}$ J. Jamnik and J. Maier, Phys. Chem. Chem. Phys. 3, 1668 (2001).

${ }^{34}$ C.-T. Sah, Fundamentals of Solid State Electronics (World Scientific, Singapore, 1991)

${ }^{35}$ C. E. D. Chidsey and R. W. Murray, J. Phys. Chem. 90, 1479 (1986).

${ }^{36}$ J. Bisquert, Electrochim. Acta 47, 2435 (2002).

${ }^{37}$ H. Bässler, Phys. Status Solidi B 175, 15 (1993).

${ }^{38}$ J. G. Simmons and G. W. Taylor, Phys. Rev. B 4, 502 (1971).

${ }^{39}$ I. Mora-Seró and J. Bisquert, Nano Lett. 3, 945 (2003).

${ }^{40}$ T. Dittrich, I. Mora-Seró, G. Garcia-Belmonte, and J. Bisquert, Phys. Rev. B 73, 045407 (2006).

${ }^{41}$ J. A. Anta, I. Mora-Seró, T. Dittrich, and J. Bisquert, J. Phys. Chem. C 111, 13997 (2007).

${ }^{42}$ J. Backholm, P. Georén, and G. A. Niklasson, J. Appl. Phys. 103, 023702 (2008).

${ }^{43}$ F. Bardé, P. L. Taberna, J. M. Tarascon, and M. R. Palacín, J. Power Sources 179, 830 (2008).

${ }^{44}$ L. M. Peter, J. Electroanal. Chem. 599, 233 (2007).

${ }^{45}$ J. Bisquert, A. Zaban, M. Greenshtein, and I. Mora-Seró, J. Am. Chem. Soc. 126, 13550 (2004).

${ }^{46}$ H. J. Snaith and L. Schmidt-Mende, Adv. Math. 19, 3187 (2007).

${ }^{47}$ J. Bisquert, J. Phys. Chem. B 108, 2323 (2004).

${ }^{48}$ J. Bisquert, J. Phys. Chem. B 106, 325 (2002).

${ }^{49}$ J. Bisquert and V. S. Vikhrenko, Electrochim. Acta 47, 3977 (2002).

${ }^{50}$ A. Pitarch, G. Garcia-Belmonte, I. Mora-Seró, and J. Bisquert, Phys. Chem. Chem. Phys. 6, 2983 (2004).

${ }^{51}$ L. Brioullin, Wave Propagation in Periodic Structures (Dover, New York, 1953). 
${ }^{52}$ F. Fabregat-Santiago, G. Garcia-Belmonte, J. Bisquert, A. Zaban, and P. Salvador, J. Phys. Chem. B 106, 334 (2002).

${ }^{53}$ J. Bisquert, G. Garcia-Belmonte, and A. Pitarch, ChemPhysChem 4, 287 (2003).

${ }^{54}$ I. Mora-Seró, J. Bisquert, F. Fabregat-Santiago, G. GarciaBelmonte, G. Zoppi, K. Durose, Y. Proskuryakov, I. Oja, A.
Belaidi, T. Dittrich, R. Tena-Zaera, A. Katty, C. Lévy-Clement, V. Barrioz, and S. J. C. Irvine, Nano Lett. 6, 640 (2006).

${ }^{55}$ F. Fabregat-Santiago, J. Bisquert, G. Garcia-Belmonte, G. Boschloo, and A. Hagfeldt, Sol. Energy Mater. Sol. Cells 87, 117 (2005). 